\title{
Spectroscopic calculations of the low-lying structure in exotic Os and W isotopes
}

\author{
K. Nomura, ${ }^{1}$ T. Otsuka,${ }^{1,2,3}$ R. Rodríguez-Guzmán, ${ }^{4}$ L. M. Robledo,${ }^{5}$ P. Sarriguren, ${ }^{4}$ P. H. Regan, ${ }^{6}$ \\ P. D. Stevenson, ${ }^{6}$ and Zs. Podolyák ${ }^{6}$ \\ ${ }^{1}$ Department of Physics, University of Tokyo, Hongo, Bunkyo-ku, Tokyo 113-0033, Japan \\ ${ }^{2}$ Center for Nuclear Study, University of Tokyo, Hongo, Bunkyo-ku, Tokyo 113-0033, Japan \\ ${ }^{3}$ National Superconducting Cyclotron Laboratory, Michigan State University, East Lansing, Michigan, USA \\ ${ }^{4}$ Instituto de Estructura de la Materia, IEM-CSIC, Serrano 123, E-28006 Madrid, Spain \\ ${ }^{5}$ Departamento de Física Teórica, Universidad Autónoma de Madrid, E-28049 Madrid, Spain \\ ${ }^{6}$ Department of Physics, University of Surrey, Guildford GU2 7XH, United Kingdom
}

(Received 11 March 2011; published 9 May 2011)

\begin{abstract}
Structural evolution in neutron-rich Os and $\mathrm{W}$ isotopes is investigated in terms of the interacting boson model (IBM) Hamiltonian determined by (constrained) Hartree-Fock-Bogoliubov calculations with the Gogny-D1S energy density functional (EDF). The interaction strengths of the IBM Hamiltonian are produced by mapping the potential energy surface (PES) of the Gogny-EDF with quadrupole degrees of freedom onto the corresponding PES of the IBM system. We examine the prolate-to-oblate shape/phase transition which is predicted to take place in this region as a function of neutron number $N$ within the considered $\mathrm{Os}$ and $\mathrm{W}$ isotopic chains. The onset of this transition is found to be more rapid compared to the neighboring Pt isotopes. The calculations also allow the prediction of spectroscopic variables (excited state energies and reduced transition probabilities) which are presented for the neutron-rich ${ }^{192,194,196} \mathrm{~W}$ nuclei, for which there is only very limited experimental data available to date.
\end{abstract}

DOI: 10.1103/PhysRevC.83.054303

\section{INTRODUCTION}

Quadrupole collectivity has long been understood as one of the most basic, yet prominent, aspects of nuclear structure $[1,2]$. Nuclei are quantum many-body systems exhibiting collective properties associated with a distinct shape of the mean field, which can be represented by a geometrical surface. Quadrupole collectivity can then be understood as a quadrupole-shaped deformation of the nuclear surface whose magnitude depends on the number of valence nucleons, and has been shown to exhibit remarkable regularities in spectroscopic observables, such as the excitation energy of the $2_{1}^{+}$state and the ratio $E_{4_{1}^{+}} / E_{2_{1}^{+}}$. Also evident are stunning shape/phase transitions at specific nucleon numbers where the collective nature of the quantal nuclear system can be well described as a phase transition between (for example) quadrupole vibrational and statically (quadrupole) deformed potentials [2,3].

The underlying multifermion dynamics of such nuclei however, is so complex that its microscopic understanding still continues to be a theme of major interest in nuclear structure physics research. Mean-field studies, based on Skyrme [4-6] and Gogny [7] as well as relativistic [4,8] energy density functionals (EDFs), provide reasonable descriptions of various nuclear properties, such as masses, charge radii, mass density distributions, and surface deformations, over a wide range of neutron and proton numbers [2,4]. Such mean-field models, with their intrinsic spontaneous symmetry breaking mechanism, are highly relevant to understanding the microscopy of the nuclear quadrupole deformation and, therefore, have been used as a starting point for predictions relevant for future nuclear spectroscopic investigations in exotic nuclei [2,4,9-11].

Phenomenological studies using the interacting boson model (IBM) [12] have enjoyed significant success in
PACS number(s): 21.10.Re, 21.60.Ev, 21.60.Fw, 21.60.Jz

describing the low-lying quadrupole collective states of medium-mass and heavy nuclei. The merit of the IBM lies in its simplicity, such that, based on group theory, the highly complicated multifermion dynamics of surface deformation can be simulated by simple, effective bosonic degrees of freedom, which correspond to (collective) pairs of valence nucleons [13]. In addition to its success in reproducing a large amount of experimental data on low-lying collective nuclear states in heavy nuclei, the microscopic derivation of the IBM Hamiltonian has also been extensively studied [13-15]. In particular, a way of deriving the interaction strengths of an IBM Hamiltonian has been proposed recently [16]. This method is based on simulating the potential energy surface (PES) of a given EDF by the corresponding IBM PES. The IBM parameters are then derived as functions of the nucleon number using the wavelet analysis method [17]. In this way, the universality of the nuclear EDF and the simplicity of the IBM can be combined, thereby, allowing the calculations to predict directly measurable spectroscopic observables, such as excitation energies and electromagnetic transition rates between specific states. A number of spectroscopic calculations have been carried out using this method for the $\mathrm{Ru}, \mathrm{Pd}, \mathrm{Ba}$, $\mathrm{Xe}$, and $\mathrm{Sm}$ isotopic chains, as well as theoretical predictions on $N>126$ Os-W nuclei using the Skyrme EDF [17].

The neutron-rich W, Os, and Pt nuclei with $A \sim 190-200$ exhibit a very challenging structural evolution, which has already been extensively studied [18-25]. As originally pointed out in [19] the ratio $E_{4_{1}^{+}} / E_{2_{1}^{+}}$in ${ }^{190} \mathrm{~W}$ is anomalously small compared with the one in neighboring isotopes. The most recent experimental data on the neutron-rich tungsten chain from ${ }^{188,190,192} \mathrm{~W}[18,21,23]$ all suggest a change from a well deformed, axially symmetric prolate shape for lighter tungsten isotopes to a more $\gamma$-soft system for ${ }^{190} \mathrm{~W}$. This 
transition from a prolate to very $\gamma$-soft system for neutron number $N=116$ (i.e., for ${ }^{190} \mathrm{~W}$ ) is consistent with the recent observation of the second $2^{+}$state in ${ }^{190} \mathrm{~W}$ which appears to lie lower than the yrast $4^{+}$in this nucleus [21]. The neutron-rich nature of the heavier $\mathrm{W}$ and Os nuclei makes them experimentally challenging to study. However, in recent years, there has been some progress made in their structural investigation following multinucleon transfer [18,23,24] and isomer and/or $\beta$-delayed $\gamma$-ray spectroscopy following projectile fragmentation reactions $[19,21,22]$. The current experimental information is limited to the yrast sequence in ${ }^{190} \mathrm{~W}[21,23]$ and the identification of the $2_{1}^{+}$state in ${ }^{192} \mathrm{~W}$ [21]. It is interesting to note that the yrast $2^{+}$states in the $N=116$ isotones ${ }^{190} \mathrm{~W}$ and ${ }^{192}$ Os have almost identical energies $(\sim 206 \mathrm{keV})$, as do the $N=118$ isotones ${ }^{192} \mathrm{~W}$ and ${ }^{194}$ Os $(\sim 218 \mathrm{keV})$.

On the theoretical side, mean-field calculations have been performed which predict the shapes of these systems both with (e.g., [26]) and without (see, e.g., Refs. [27,28], and references therein) the assumption of axial symmetry in the nuclear mean field. The IBM has also been applied to fit the spectral properties of $\mathrm{W}$ isotopes in a phenomenological way [29]. More recently, spectroscopic calculations have been carried out [30] to describe the structural evolution in Pt isotopes with the Gogny-D1S EDF [31]. In this paper, we review the current spectroscopy relevant to the prolateto-oblate shape/phase transition in neutron-rich Os and $\mathrm{W}$ isotopes. We also report the predicted excitation spectra and the transition probabilities on the neutron-rich $\mathrm{Os}$ and $\mathrm{W}$ nuclei. The spectroscopic calculations have been carried out in terms of the IBM Hamiltonian derived by mapping (constrained) Hartree-Fock-Bogoliubov (HFB) calculations, based on the Gogny-D1S EDF, using a similar technique as in [30].

\section{THEORETICAL PROCEDURES}

We begin with the calculation of the PES in terms of the (constrained) HFB method using the Gogny-D1S EDF. The solution of the HFB equations, leading to the set of vacua $\left|\Phi_{\mathrm{HFB}}\right\rangle$, is based on the equivalence of the HFB with a minimization problem which is solved using the gradient method [28]. In agreement with the fitting protocol of the force, the kinetic energy of the center-of-mass motion is subtracted from the Routhian to be minimized, in order to ensure that the center of mass is kept at rest. The exchange Coulomb energy is considered in the Slater approximation, and the contribution of the Coulomb interaction to the pairing field is neglected. The HFB quasiparticle operators are expanded in a harmonic oscillator (HO) basis having enough number of shells (i.e., $N_{\text {shell }}=13$ major shells) to grant convergence for all values of the mass quadrupole operators and for all the nuclei studied. The constraint is imposed on the average values of the mass quadrupole operators $\hat{Q}_{20}=\frac{1}{2}\left(2 z^{2}-x^{2}-y^{2}\right)$ and $\hat{Q}_{22}=\frac{\sqrt{3}}{2}\left(x^{2}-y^{2}\right)$ to the desired deformation values $Q_{20}=\left\langle\Phi_{\mathrm{HFB}}\left|\hat{Q}_{20}\right| \Phi_{\mathrm{HFB}}\right\rangle$ and $Q_{22}=\left\langle\Phi_{\mathrm{HFB}}\left|\hat{Q}_{22}\right| \Phi_{\mathrm{HFB}}\right\rangle$. In Refs. [27,28], the $Q-\gamma$ energy contour plots with $Q=$ $\sqrt{Q_{20}^{2}+Q_{22}^{2}}$ and $\tan \gamma=Q_{22} / Q_{20}$ have been used to study the (mean-field) evolution of the ground-state shapes in $\mathrm{Pt}$ nuclei. Alternatively, one could also consider the $\beta-\gamma$ representation in which the quadrupole deformation parameter $\beta$ is written [28] in terms of $Q$ as $\beta=\sqrt{\frac{4 \pi}{5}} \frac{Q}{A\left\langle r^{2}\right\rangle}$, where $\left\langle r^{2}\right\rangle$ represents the mean squared radius evaluated with the corresponding HFB state $\left|\Phi_{\mathrm{HFB}}\right\rangle$. The set of constrained HFB calculations provides the Gogny-D1S PES, i.e., the total HFB energies $E_{\mathrm{HFB}}(\beta, \gamma)$.

For the bosonic mapping, we use the IBM-2, comprised of independent $L=0^{+}, 2^{+}$proton $\left(s_{\pi}, d_{\pi}\right)$ and neutron $\left(s_{\nu}\right.$, $d_{\nu}$ ) bosons. The number of proton (neutron) bosons, denoted by $n_{\pi}\left(n_{v}\right)$, is equal to half of the number of valence protons (neutrons), assuming the usual magic-number shell gaps at $Z=50$ and 82, and $N=82$ and 126. We adopt the standard IBM-2 Hamiltonian [16,17,30]

$$
\hat{H}_{\mathrm{IBM}}=\epsilon\left(\hat{n}_{d \pi}+\hat{n}_{d v}\right)+\kappa \hat{Q}_{\pi} \cdot \hat{Q}_{\nu},
$$

where $\hat{n}_{d \rho}=d_{\rho}^{\dagger} \cdot \tilde{d}_{\rho}$ and $\hat{Q}_{\rho}=\left[s_{\rho}^{\dagger} \tilde{d}_{\rho}+d_{\rho}^{\dagger} \tilde{s}_{\rho}\right]^{(2)}+\chi_{\rho}\left[d_{\rho}^{\dagger} \tilde{d}_{\rho}\right]^{(2)}$ with $\rho=\pi, v$. The bosonic PES is represented by the expectation value of $\hat{H}_{\mathrm{IBM}}$ in the boson coherent state [32], given by

$$
|\Phi\rangle \propto \prod_{\rho=\pi, \nu}\left[s_{\rho}^{\dagger}+\sum_{\mu=0, \pm 2} \alpha_{\rho \mu} d_{\rho \mu}^{\dagger}\right]^{n_{\rho}}|0\rangle,
$$

where $|0\rangle$ stands for the boson vacuum (i.e., inert core) and the coefficients $\alpha$ 's are expressed as $\alpha_{\rho 0}=\beta_{\rho} \cos \gamma_{\rho}, \alpha_{\rho \pm 1}=0$, and $\alpha_{\rho \pm 2}=\frac{1}{\sqrt{2}} \beta_{\rho} \sin \gamma_{\rho}$. The intrinsic shape of the nucleus is then described in terms of the (axially symmetric) deformation $\beta_{\rho}$ and the (triaxial) deformation $\gamma_{\rho}$. The IBM PES reads $[16,17]$

$$
\begin{aligned}
E_{\mathrm{IBM}}\left(\beta_{B}, \gamma_{B}\right)= & \frac{\epsilon\left(n_{\pi}+n_{v}\right) \beta_{B}^{2}}{1+\beta_{B}^{2}}+n_{\pi} n_{\nu} \kappa \frac{\beta_{B}^{2}}{\left(1+\beta_{B}^{2}\right)^{2}} \\
& \times\left[4-2 \sqrt{\frac{2}{7}}\left(\chi_{\pi}+\chi_{\nu}\right) \beta_{B} \cos 3 \gamma_{B}\right. \\
& \left.+\frac{2}{7} \chi_{\pi} \chi_{\nu} \beta_{B}^{2}\right],
\end{aligned}
$$

where $\beta_{\pi}=\beta_{\nu} \equiv \beta_{B}$ and $\gamma_{\pi}=\gamma_{\nu} \equiv \gamma_{B}$ is assumed for simplicity $[16,17]$. We also assume the proportionality, i.e., $\beta_{B}=C_{\beta} \beta$, where $C_{\beta}$ is a numerical coefficient, and $\gamma_{B}=\gamma$ $[16,17]$. In this context, the variables $\beta_{B}$ and $\gamma_{B}$ represent the boson images of the (fermion) deformation parameters $(\beta$, $\gamma)$. A point on the HFB PES, $(\beta, \gamma)$, within an energy range relevant for the considered low-lying quadrupole collective states, is mapped onto the corresponding point on the IBM PES, $\left(\beta_{B}, \gamma_{B}\right)$. The $\epsilon, \kappa, \chi_{\pi, v}$, and $C_{\beta}$ values are fixed for a given nucleus by drawing the IBM PES so that the surface topology of the corresponding HFB PES is reproduced. This is done unambiguously by means of the recently developed procedure [17] using the wavelet transform [33].

Note that we compare the total energies $E_{\mathrm{HFB}}(\beta, \gamma)$ and $E_{\mathrm{IBM}}(\beta, \gamma)$. By reproducing the HFB PES as much as possible, effects of both vibrational and rotational kinetic energies, similar to those introduced when solving a five-dimensional (5D) collective Bohr Hamiltonian (see, for example, Refs. [9-11]), should be included in the boson systems. For a 
large deformation, however, the rotational response, i.e., the response to cranking, differs significantly between nucleon and boson systems, resulting in the deviation of the IBM rotational spectra from fermionic ones. This deviation could be corrected by introducing an additional rotational kineticlike term, i.e., the so-called $L \cdot L$ term [34] in the IBM Hamiltonian [35]. This problem does not show up in the present work where only the moderately deformed nuclei are concerned, and, thus, we neglect the $L \cdot L$ term in the boson Hamiltonian of Eq. (1).

We also note that to what extent the present mapping procedure mimics the solution of a 5D Bohr Hamiltonian is an interesting open question, which may be partly answered by looking at how reasonably our results compare with the ones of the 5D Hamiltonian and the available experimental data.

\section{MAPPED IBM PES AND THE DERIVED PARAMETERS}

Figure 1 shows the mapped IBM PESs for ${ }^{190-196}$ Os and ${ }^{188-194} \mathrm{~W}$ nuclei up to $2 \mathrm{MeV}$ excitation from the energy minimum. The corresponding HFB PESs have been reported in Fig. 3 of Ref. [28]. The PESs for both Os and W nuclei show similar tendencies. There are quantitative differences between the $\mathrm{Pt}$ and $\mathrm{Os}-\mathrm{W}$ isotopic chains, namely, that the

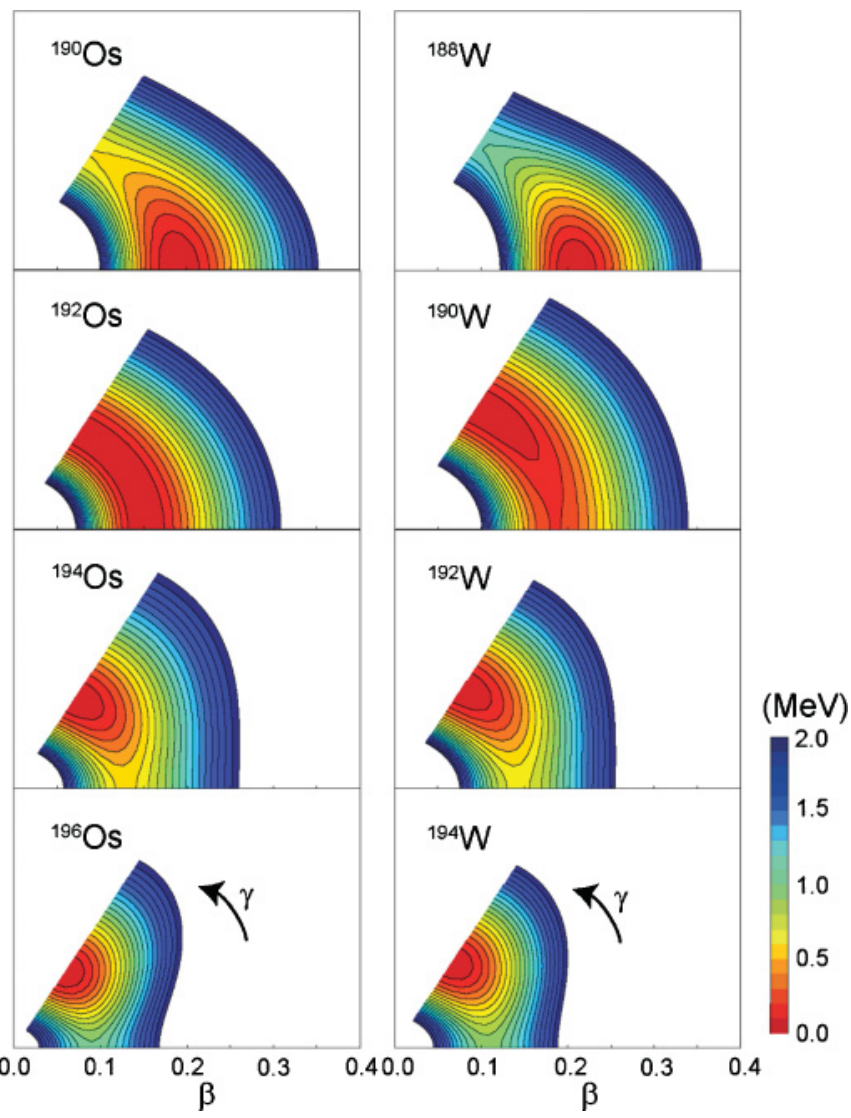

FIG. 1. (Color online) Mapped potential energy surfaces (PESs) for ${ }^{190-196} \mathrm{Os}$ and ${ }^{188-194} \mathrm{~W}$ nuclei up to $2 \mathrm{MeV}$ excitation from the energy minimum within the ranges $0^{\circ} \leqslant \gamma \leqslant 60^{\circ}$. The PESs are shown in terms of the fermionic deformation parameters $\beta\left(=\beta_{B} / C_{\beta}\right)$ and $\gamma\left(=\gamma_{B}\right)$. topology of the PES changes more slowly in the former [30], compared to the latter in Fig. 1. An (almost) axially symmetric, oblate minima is observed in Pt nuclei with $N=114-120$, and shallow triaxiality for $N=110$ and 112 [28,30]. On the other hand, the Os and $\mathrm{W}$ isotopes are predicted to have the corresponding oblate minima only for $N=118$ and 120, with a more rapid change to axially symmetric prolate deformation for $N \leqslant 114$. Indeed, shallow triaxiality (i.e., $\gamma$ softness) appears only around $N=116$ for both Os and W nuclei [28]. The corresponding mapped IBM PESs reproduce these trends of the HFB PESs of [28] well, whereas the location of the minimum in the IBM PES differs from that of the HFB PES of Ref. [28] in some nuclei as the present IBM PES of Eq. (3) does not produce a triaxial minimum. The mapped PES for the $N=116$ isotone, ${ }^{192}$ Os is predicted to be very flat along the $\gamma$ direction. Similarly, the IBM PES for ${ }^{190} \mathrm{~W}$ is also very flat, with the global energy minimum corresponding to a quadrupole deformation of $\beta \sim 0.15$ on the oblate side. This flatness is the consequence of the $\chi_{\pi}$ and $\chi_{\nu}$ parameter values, such that their sum is close to 0 . Comparing Os and $\mathrm{W}$ isotopes with the same neutron number, the $\mathrm{W}$ nuclei are generally steeper in both $\beta$ and $\gamma$ directions than the corresponding Os isotone. A similar trend is also observed in the corresponding HFB PESs [28].
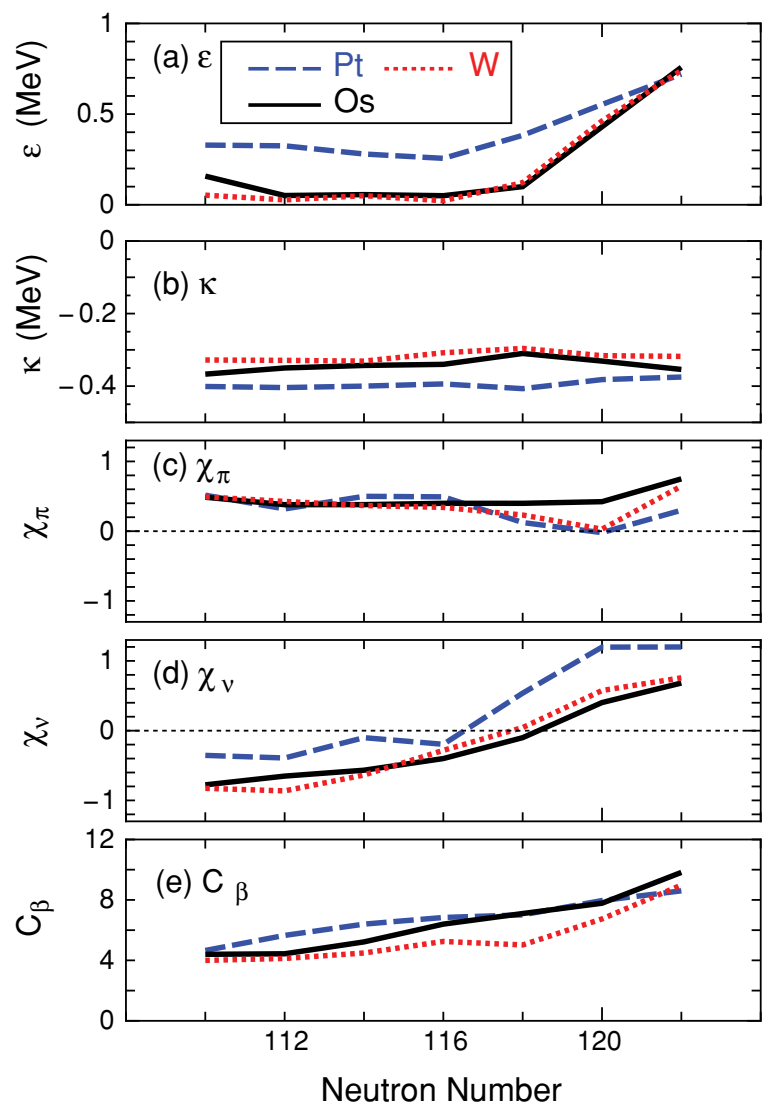

FIG. 2. (Color online) Derived IBM parameter values for the considered Os and W nuclei, represented by solid and dotted curves, respectively, as functions of $N$. Results for Pt isotopes taken from Ref. [30] are also depicted for comparison. 
Figure 2 shows the evolution of the derived IBM parameters for the considered Os and W nuclei as functions of the neutron number $N$. The parameter values for Pt nuclei, taken from Ref. [30], are also shown for comparison. There are significant differences in quantitative details of the derived IBM parameter values between Os-W and Pt nuclei. In particular, the values of the parameter $\epsilon$ in Fig. 2(a) for Os and W nuclei are rather small in the region away from the shell closure as compared to Pt nuclei. In Fig. 2(b), the magnitude of the parameter $\kappa$ is smaller than the analogous results for the Pt isotopes. The behavior of the parameters $\epsilon$ and $\kappa$ is reflective of the HFB PESs for Os and W nuclei being somewhat steeper in the $\beta$ degree of freedom compared to the Pt isotopes, as discussed in Ref. [28]. The $\chi_{\pi, v}$ parameters in Figs. 2(c) and 2(d) (as well as their sum) behave similarly to those of Pt nuclei. For both $N=110,112$, the sum is almost 0 in Pt isotopes, while it is small for Os and $\mathrm{W}$ ones, but has a negative sign. This indicates a weak prolate deformation in the latter as seen in Fig. 1. In other words, the $\gamma$-soft structure is rather sustained in these $\mathrm{Pt}$ isotopes, but it is not for the corresponding Os and $\mathrm{W}$ isotopes. As in Fig. 2(e), the scale parameter $C_{\beta}$ in the present case behaves similarly as for the Pt nuclei with about the same order of magnitude.

\section{CALCULATION OF THE ENERGY SPECTRA AND $B(E 2)$ VALUES}

Using the derived parameters, we calculate excitation spectra and reduced $E 2$ transition probabilities $B(E 2)$. The Hamiltonian of Eq. (1) is diagonalized by using the code NPBOS [36].

Figure 3 shows ground-state (g.s.) band and the quasi- $\gamma$ bandhead $2_{2}^{+}$(denoted by $2_{\gamma}^{+}$) energies for Os and W isotopes. In general, the calculated results follow the experimental trends reasonably well, particularly for $2_{1}^{+}$energy. What is of interest
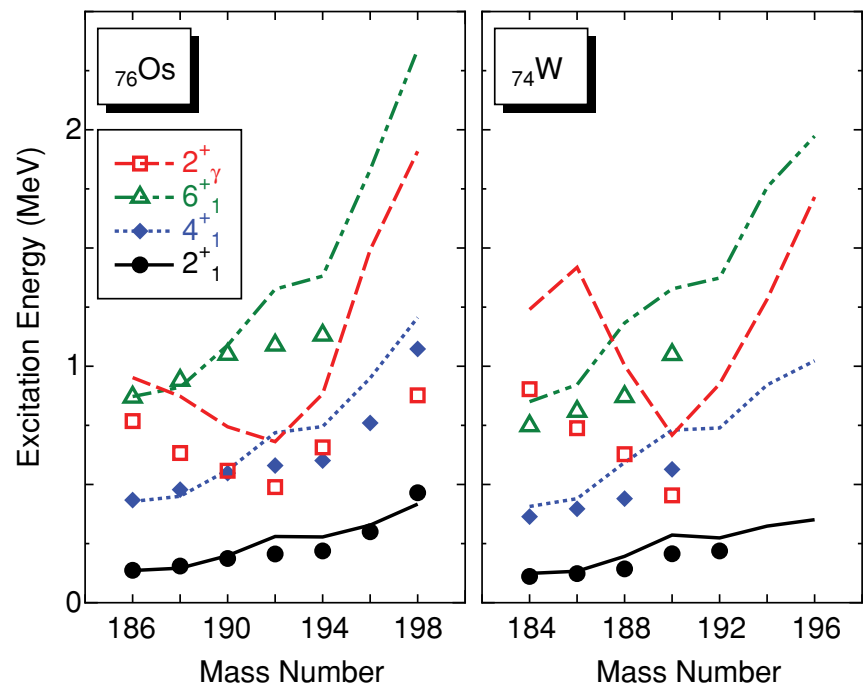

FIG. 3. (Color online) Low-lying g.s. band and quasi- $\gamma$ bandhead $\left(2_{\gamma}^{+}\right)$energies (curves) for ${ }^{186-198}$ Os and ${ }^{184-196} \mathrm{~W}$ nuclei. Experimental data (symbols) are taken from Refs. [21,37,38]. GognyD1S EDF is used. in Fig. 3 is the behavior of the $2_{\gamma}^{+}$energy, exhibiting a kink for both ${ }^{192} \mathrm{Os}$ and ${ }^{190} \mathrm{~W}$. The experimental $2_{\gamma}^{+}$energy in ${ }^{192} \mathrm{Os}$ is lower than the $4_{1}^{+}$one. This is an evidence that the $A=192$ nucleus is the most $\gamma$ unstable one among other Os isotopes. The present calculation follows the trend for Os isotopes well, and predicts a similar one for $\mathrm{W}$ isotopes exhibiting, however, more rapid change as a function of $N$. The location of the $2_{\gamma}^{+}$ state for ${ }^{196} \mathrm{Os}\left({ }^{192} \mathrm{~W}\right)$ has not yet been fixed experimentally, but the present calculations suggest that the $4_{1}^{+}$state is lower than the $2_{\gamma}^{+}$one in both ${ }^{196} \mathrm{Os}$ and ${ }^{192} \mathrm{~W}$. The calculated $2_{\gamma}^{+}$ energy is generally higher than the experimental one, whereas the qualitative feature of the experimental level is reproduced well.

Now, we turn to the analysis of $B(E 2)$ systematics, relevant to the considered low-lying states. The $B(E 2)$ value is given by

$$
B\left(E 2 ; J \rightarrow J^{\prime}\right)=\frac{1}{2 J+1}\left|\left\langle J^{\prime}|| \hat{T}^{(E 2)} \| J\right\rangle\right|^{2},
$$

where $J$ and $J^{\prime}$ are the angular momenta for the initial and final states, respectively. The $E 2$ transition operator $\hat{T}^{(E 2)}$ is given by $\hat{T}^{(E 2)}=e_{\pi} \hat{Q}_{\pi}+e_{\nu} \hat{Q}_{\nu}$. Here, $e_{\pi}$ and $e_{\nu}$ stand for the boson effective charges. These effective charges should be in principle determined not at the mean-field level, but rather by some treatment taking into account effects beyond the mean field, such as core polarization. This is, however,

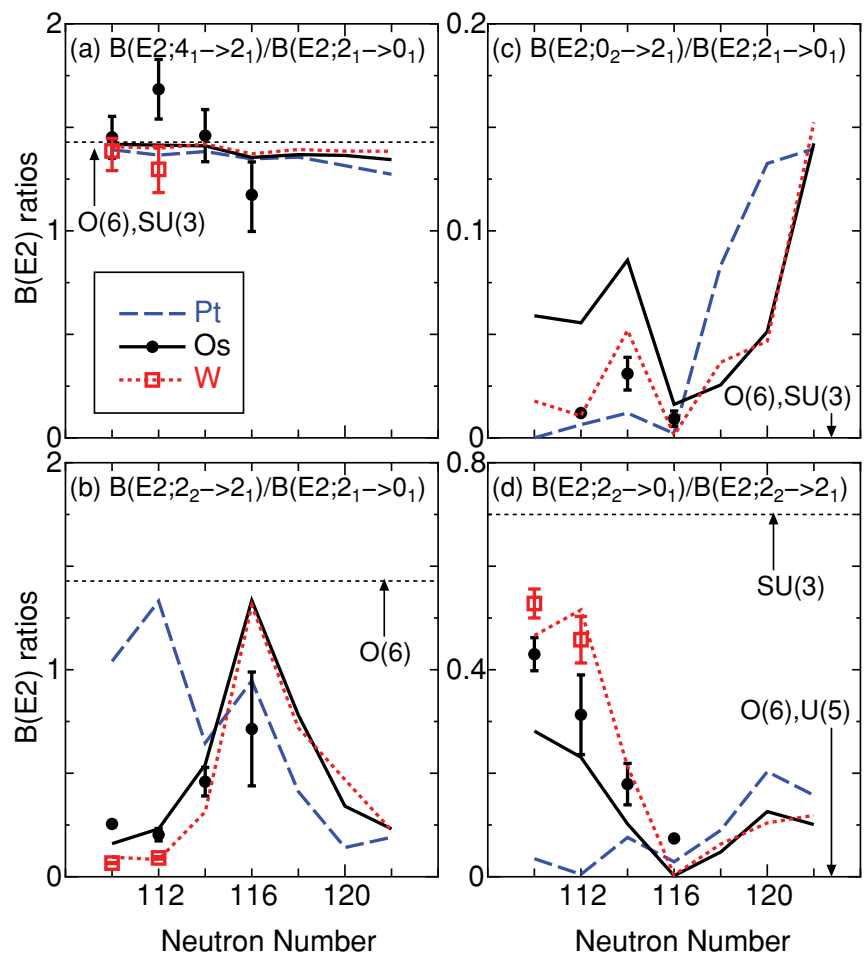

FIG. 4. (Color online) Theoretical (curves) and experimental (symbols with error bars) [39] $B(E 2)$ ratios for Os and $\mathrm{W}$ isotopes as functions of $N$. Theoretical results for Pt isotopes taken from Ref. [30] are also depicted as dashed curves, for comparison. Gogny-D1S EDF is used. 
beyond the scope of the current framework and may need to be investigated in the future. In what follows, we assume $e_{\pi}=e_{v}$, for simplicity, and discuss ratios of $B(E 2)$ s rather than their absolute values and the quadrupole moments for the corresponding excited states. Note that the $B(E 2)$ ratio at each dynamical symmetry limit, shown below, means the one with infinite boson number [12].

From Fig. 4(a), we observe that the ratio $R_{1} \equiv B\left(E 2 ; 4_{1}^{+} \rightarrow\right.$ $\left.2_{1}^{+}\right) / B\left(E 2 ; 2_{1}^{+} \rightarrow 0_{1}^{+}\right)$does not change much, being close to its $\mathrm{O}(6)$ limit of IBM $10 / 7$ [which is also the $\mathrm{SU}(3)$ limit of $R_{1}$ ]. This trend persists for $N \geqslant 118$ where there is currently no available data. The ratio $R_{2} \equiv B\left(E 2 ; 2_{2}^{+} \rightarrow\right.$ $\left.2_{1}^{+}\right) / B\left(E 2 ; 2_{1}^{+} \rightarrow 0_{1}^{+}\right)$, shown in Fig. 4(b), is of particular interest as one can observe a significant difference in its value for the Pt and Os-W isotopes. The magnitude of the $R_{2}$ ratio is arguably the most appropriate and sensitive fingerprint for $\gamma$ softness [30]. The $R_{2}$ values for both $\mathrm{Pt}$ and Os-W are relatively large and close to the $\mathrm{O}(6)$ limit $(=10 / 7)$ for $N=114-118$, where the nuclei show notable $\gamma$ instability. For Pt nuclei, this trend persists even for $N \leqslant 112$, while smaller values are suggested for Os and $\mathrm{W}$ nuclei. These differences between the $\mathrm{Pt}$ and Os-W chains reflect the difference in the topology of the PES. The results for Os nuclei follow the experimental trend, which increases for $N=110-116$. The present calculation for Os nuclei suggests the decrease of the $R_{2}$ value for $N \geqslant 118$, which corresponds to a suppression of $\gamma$ softness. The ratio $R_{3} \equiv B\left(E 2 ; 0_{2}^{+} \rightarrow 2_{1}^{+}\right) / B\left(E 2 ; 2_{1}^{+} \rightarrow 0_{1}^{+}\right)$ in Fig. 4(c) generally has a predicted value which is rather small, being close to 0 [corresponding to the $\mathrm{O}(6)$ and $\mathrm{SU}(3)$ limits], as compared to $R_{1}$ and $R_{2}$ values. Note that the scale of the vertical axis in Fig. 4(c) is different from those of Figs. 4(a) and 4(b). No rapid change with $N$ is seen for $R_{3}$ as in $R_{2}$. Nevertheless, we should note the quantitative differences between the $\mathrm{Pt}$ and the Os-W nuclei. The branching ratio $R_{4} \equiv B\left(E 2 ; 2_{2}^{+} \rightarrow 0_{1}^{+}\right) / B\left(E 2 ; 2_{2}^{+} \rightarrow 2_{1}^{+}\right)$in Fig. $4(\mathrm{~d})$ for Os follows the experimental trend for $N=110-116$. The decrease of the $R_{4}$ value from $N=110$, close to $7 / 10$ [SU(3) limit], toward $N=116$, close to $0[\mathrm{O}(6)$ and $\mathrm{U}(5)$ limit], reflects the corresponding structural evolution. The $R_{4}$ value for the $\mathrm{Pt}$ chain is close to 0 , while for the Os-W chains, there is a significant change at $N=116$. For the $\mathrm{W}$ nuclei, the ratio $R_{4}$ increases more rapidly than for the Os chain from $N=116$ to 112 . Earlier phenomenological studies suggested a similar increase [29].

Finally, we present in Fig. 5 the level schemes corresponding to the neutron-rich nuclei ${ }^{190,192,194,196}$ Os and ${ }_{190,192,194} \mathrm{~W}$ taken as representative samples. For ${ }^{190,192} \mathrm{Os}$, for which there are significant experimental data, not only the g.s. band but also both the quasi- $\gamma$-bandhead $2_{\gamma}^{+}$and the quasi- $\beta$-bandhead $0_{2}^{+}$(denoted by $0_{\beta}^{+}$) energies are reproduced quite well by the current calculations, although the detailed "in-band" energy staggering looks different between the
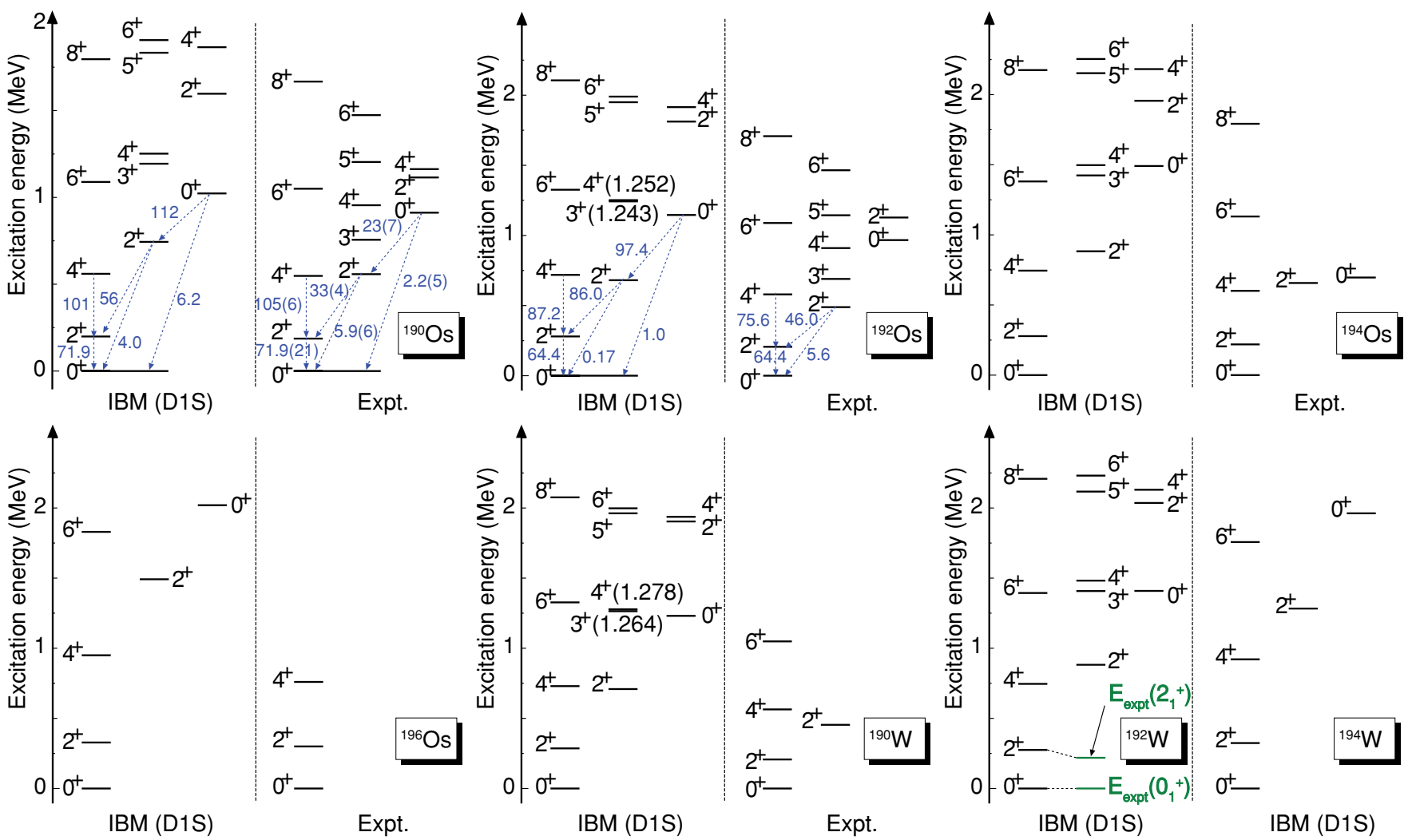

FIG. 5. (Color online) Level schemes for ${ }^{190,192,194,196}$ Os and ${ }^{190,192,194} \mathrm{~W}$ nuclei. The theoretical $B(E 2)$ values (in Weisskopf units) for ${ }^{190,192}$ Os are normalized to the experimental [39] $B\left(E 2 ; 2_{1}^{+} \rightarrow 0_{1}^{+}\right)$value. Calculated $3_{1}^{+}$and $4_{2}^{+}$energies for ${ }^{192} \mathrm{Os}$ and ${ }^{190} \mathrm{~W}$ are shown (in MeV units) in the parentheses because, otherwise, these energies look identical. Note that, for ${ }^{192} \mathrm{~W}$, experimental data are shown in the same panel as theoretical ones. Gogny-D1S EDF is used. 
calculated and the experimental levels. The calculated $B(E 2)$ values for ${ }^{190,192}$ Os have been normalized to the experimental [39] $B\left(E 2 ; 2_{1}^{+} \rightarrow 0_{1}^{+}\right)$value. Some algebraic feature is also apparent in the calculated results. The $\Delta \tau= \pm 1$ rule for the $E 2$ decay pattern at the $\mathrm{O}(6)$ limit [12] [i.e., the dominance of $2_{2}^{+} \rightarrow 2_{1}^{+}\left(0_{2}^{+} \rightarrow 2_{2}^{+}\right)$over $\left.2_{2}^{+} \rightarrow 0_{1}^{+}\left(0_{2}^{+} \rightarrow 2_{1}^{+}\right)\right]$in the present calculation also compares well with the experimental decay pattern.

The experimental value of the $2_{1}^{+}$energy for ${ }^{192}$ Os is very close to that of its isotone ${ }^{190} \mathrm{~W}$ (i.e., $E \approx 207 \mathrm{keV}$ ). Also, the excitations energies of the $2_{1}^{+}$levels in these isotones are also quite similar to each other. The present calculations reproduce this overall trend well. In fact, the calculated $E\left(2_{1}^{+}\right)=0.280(0.278)$ and $0.286(0.274) \mathrm{MeV}$ for ${ }^{192} \mathrm{Os}$ $\left({ }^{194} \mathrm{Os}\right)$ and ${ }^{190} \mathrm{~W}\left({ }^{192} \mathrm{~W}\right)$ nuclei, respectively. For ${ }^{192} \mathrm{Os}$ and ${ }^{190} \mathrm{~W}$ nuclei, the calculated g.s. band energies are rather stretched, and the $2_{\gamma}^{+}$energies are in good agreement with the respective experimental data. In the calculated quasi- $\gamma$ band of ${ }^{190}$ Os and ${ }^{192}$ Os nuclei, one observes a staggering as $2_{\gamma}^{+}\left(3_{\gamma}^{+} 4_{\gamma}^{+}\right)\left(5_{\gamma}^{+} 6_{\gamma}^{+}\right), \ldots$, etc. By contrast, the experimental energy spacing shows a more regular pattern. This deviation may be related to the topology of the mapped IBM PES in Fig. 1, which is flat in $\gamma$ direction, while the corresponding Gogny-D1S PES exhibits shallow triaxial minimum [28]. In the future, some additional interaction term, such as a so-called cubic term [40], may need to be introduced in the boson Hamiltonian to correct the deviation for detailed structure of quasi- $\gamma$ band.

For ${ }^{194,196}$ Os nuclei, the predicted $2_{1}^{+}$and $4_{1}^{+}$energies reproduce the experimental ones. The quasi- $\beta$-bandhead energy for ${ }^{194}$ Os in the present calculation is notably larger than the experimental value, which is a consequence of the peculiar topology of the Gogny-HFB PES, which exhibit a pronounced oblate minimum with a relatively small deformation. This results in the larger value of the parameter $\kappa$ than the one in the IBM phenomenology [29] which would give good agreement for the excited $0^{+}$energies. The positions of the $2_{\gamma}^{+}$and the $0_{\beta}^{+}$ energies for ${ }^{196}$ Os are predicted to lie below and beyond the $6_{1}^{+}$level, respectively. For the exotic ${ }^{192} \mathrm{~W}$ and ${ }^{194} \mathrm{~W}$ nuclei, the present calculation suggests a quite similar level pattern to their respective isotones, ${ }^{194}$ Os and ${ }^{196}$ Os.

\section{SUMMARY}

To summarize, we have presented the predicted excitation spectra and $B(E 2)$ ratios of exotic Os and $\mathrm{W}$ isotopes with $N=114-120$. Spectroscopic calculations have been carried out in terms of the IBM Hamiltonian constructed by the constrained HFB calculations with the Gogny-D1S EDF. We have examined the prolate-to-oblate shape/phase transition as functions of neutron number $N$ in the considered isotopic chains. The experimental trends of not only g.s.-band energies, but also the quasi- $\gamma$-bandhead $2_{\gamma}^{+}$energy for Os isotopes, are reproduced well, suggesting that the $N=116$ nucleus is the softest in $\gamma$. A similar pattern is predicted in $\mathrm{W}$ isotopes, while the evolution of levels appears to occur more rapidly in $\mathrm{W}$ than in Os. Interestingly enough, all these results reflect to a good extent the results of the underlying microscopic Gogny-HFB calculations. Lastly, let us comment on the form of the boson Hamiltonian in Eq. (1). While this form may be rather simple, it determines the basic topology of the PES, and is supposed to be the most relevant for the description of the low-lying structure at the present stage. On the other hand, the IBM-2 phenomenology considers additional interaction terms as compared to those in Eq. (1). Some of these terms have a minor effect, but others might affect the spectroscopic results in quantitative details as suggested in the structure of quasi- $\gamma$ bands in Fig. 5. It should be then very interesting to study in the future which parts of a more general boson Hamiltonian are crucial, as well as how they affect the spectroscopic properties quantitatively.

\section{ACKNOWLEDGMENTS}

This work was supported in part by grants-in-aid for Scientific Research (A) 20244022 and No. 217368 and by the JSPS Core-to-Core Program EFES. K. N. is supported by the JSPS. P. H. R., Z. P., and P. D. S. acknowledge financial support from STFC(UK). The work of L. M. R., P. S., and R. R. has been supported by MICINN (Spain) under research Grants No. FIS2008-01301, No. FPA2009-08958, and No. FIS200907277, as well as by Consolider-Ingenio 2010 Programs CPAN CSD2007-00042 and MULTIDARK CSD2009-00064. R. R. thanks Professor P. M. Walker, Professor J. Äysto, and Professor R. Julin for encouraging discussions.
[1] A. Bohr and B. R. Mottelson, Nuclear Structure, 1st ed. (Benjamin, New York, 1975), Vols. I and II.

[2] P. Ring and P. Schuck, The Nuclear Many-Body Problem (Springer, Berlin, 1980).

[3] P. Cejnar, J. Jolie, and R. F. Casten, Rev. Mod. Phys. 82, 2155 (2010).

[4] M. Bender, P.-H. Heenen, and P.-G. Reinhard, Rev. Mod. Phys. 75, 121 (2003).

[5] T. H. R. Skyrme, Nucl. Phys. 9, 615 (1959).

[6] D. Vautherin and D. M. Brink, Phys. Rev. C 5, 626 (1972).

[7] J. Decharge, M. Girod, and D. Gogny, Phys. Lett. B 55, 361 (1975).

[8] D. Vretenar, A. V. Afanasjev, G. A. Lalazissis, and P. Ring, Phys. Rep. 409, 101 (2005).
[9] P. Bonche, J. Dobaczewski, H. Flocard, P.-H. Heenen, and J. Meyer, Nucl. Phys. A 510, 466 (1990).

[10] J.-P. Delaroche, M. Girod, J. Libert, H. Goutte, S. Hilaire, S. Peru, N. Pillet, and G. F. Bertsch, Phys. Rev. C 81, 014303 (2010).

[11] Z. P. Li, T. Nikšić, D. Vretenar, and J. Meng, Phys. Rev. C 81, 034316 (2010); Z. P. Li, T. Nikšić, D. Vretenar, J. Meng, G. A. Lalazissis, and P. Ring, ibid. 79, 054301 (2009).

[12] A. Arima and F. Iachello, Phys. Rev. Lett. 35, 1069 (1975); F. Iachello and A. Arima, The Interacting Boson Model (Cambridge University Press, Cambridge, 1987).

[13] T. Otsuka, A. Arima, F. Iachello, and I. Talmi, Phys. Lett. B 76, 139 (1978); T. Otsuka, A. Arima, and F. Iachello, Nucl. Phys. A 309, 1 (1978). 
[14] T. Otsuka, in Algebraic Approaches to Nuclear Structure, edited by R. F. Casten (Harwood, Chur, 1993), p. 195.

[15] T. Mizusaki and T. Otsuka, Prog. Theor. Phys. Suppl. 125, 97 (1996).

[16] K. Nomura, N. Shimizu, and T. Otsuka, Phys Rev. Lett. 101, 142501 (2008).

[17] K. Nomura, N. Shimizu, and T. Otsuka, Phys Rev. C 81, 044307 (2010).

[18] T. Shizuma et al., Eur. Phys. J. A 30, 391 (2006).

[19] Z. Podolyák et al., Phys. Lett. B 491, 225 (2000).

[20] J. Jolie and A. Linnemann, Phys. Rev. C 68, 031301(R) (2003).

[21] N. Alkhomashi et al., Phys. Rev. C 80, 064308 (2009).

[22] P. H. Regan et al., Int. J. Mod. Phys. E 17, 8 (2008).

[23] G. J. Lane et al., Phys. Rev. C 82, 051304 (2010).

[24] C. Wheldon, J. GarcesNarro, C. J. Pearson, P. H. Regan, Z. Podolyak, D. D. Warner, P. Fallon, A. O. Macchiavelli, and M. Cromaz, Phys. Rev. C 63, 011304 (2000).

[25] P. D. Bond, R. F. Casten, D. D. Warner, and D. Horn, Phys. Lett. B 130, 167 (1983).

[26] P. D. Stevenson, M. P. Brine, Z. Podolyák, P. H. Regan, P. M. Walker, and J. R. Stone, Phys. Rev. C 72, 047303 (2005).

[27] R. Rodríguez-Guzmán, P. Sarriguren, L. M. Robledo, and J. E. García-Ramos, Phys. Rev. C 81, 024310 (2010).

[28] L. M. Robledo, R. Rodríguez-Guzmán, and P. Sarriguren, J. Phys. G: Nucl. Part. Phys. 36, 115104 (2009).
[29] P. D. Duval, and B. R. Barrett, Phys. Rev. C 23, 492 (1981).

[30] K. Nomura, T. Otsuka, R. Rodríguez-Guzmán, L. M. Robledo, and P. Sarriguren, Phys. Rev C 83, 014309 (2011).

[31] J. F. Berger, M. Girod, and D. Gogny, Nucl. Phys. A 428, 23c (1984).

[32] A. E. L. Dieperink, O. Scholten, and F. Iachello, Phys. Rev. Lett. 44, 1747 (1980).

[33] G. Kaiser, A Friendly Guide to Wavelets (Birkhäser, Boston, 1994).

[34] A. Arima and F. Iachello, Ann. Phys. (NY) 111, 201 (1978)

[35] K. Nomura, T. Otsuka, N. Shimizu, and L. Guo, arXiv:1011.1056.

[36] T. Otsuka and N. Yoshida, JAERI-M Report No. 85 (Japan Atomic Energy Research Institute, 1985).

[37] Z. Podolyák et al., Phys. Rev. C 79, 031305 (2009).

[38] Brookhaven National Nuclear Data Center (NNDC), [www.nndc.bnl.gov/].

[39] C. M. Baglin, Nucl. Data Sheets 111, 275 (2010); 99, 1 (2003); B. Singh, ibid. 95, 387 (2002); 99, 275 (2003); C. M. Baglin, ibid. 84, 717 (1998); B. Singh, ibid. 107, 1531 (2006); H. Xiaolong, ibid. 108, 1093 (2007); 110, 2533 (2007).

[40] K. Heyde, P. Van Isacker, M. Waroquier, and J. Moreau, Phys. Rev. C 29, 1420 (1984). 